\title{
Severe ileitis associated with capecitabine: Two case reports and review of the literature
}

\author{
SHING FUNG LEE, CHI LEUNG CHIANG, ANN SHING LEE, \\ FRANK CHI SHING WONG and STEWART YUK TUNG
}

\author{
Department of Clinical Oncology, Tuen Mun Hospital, Tuen Mun, Hong Kong, SAR, P.R. China
}

Received June 30, 2015; Accepted August 4, 2015

DOI: $10.3892 / \mathrm{mco} .2015 .635$

\begin{abstract}
Capecitabine is a commonly used anticancer drug, which has been associated with adverse events, including skin and gastrointestinal symptoms, such as vomiting and diarrhea. We herein present treated two rare cases of capecitabine-associated ileitis. In one of the patients, ileitis occurred during combination chemotherapy for metastatic colon cancer, despite previous good tolerance to this drug; the other patient developed ileitis following adjuvant single-agent treatment. The first case is unlike previously reported cases, in which patients had no past exposure to capecitabine. Ileitis may be severe but reversible with early diagnosis and proper supportive treatment, and patients may resume chemotherapy following capecitabine discontinuation.
\end{abstract}

\section{Introduction}

Capecitabine is an oral fluorouracil (FU) prodrug commonly used in patients with colorectal cancer, particularly as adjuvant and palliative chemotherapy in colon cancer (1). The most common gastrointestinal adverse events include nausea, vomiting and diarrhea. Capecitabine may also cause serious gastrointestinal adverse events, such as intestinal perforation or obstruction (1).

We treated 2 patients with ileitis associated with capecitabine administration. In one of the patients, ileitis occurred during palliative second-line combination chemotherapy for metastatic colon cancer, despite good tolerance to capecitabine during previous chemotherapy. A literature search identified no previous reported cases among Chinese adults who had received capecitabine as second-line chemotherapy. The other patient developed ileitis while on single-agent adjuvant therapy with capecitabine for sigmoid colon cancer. The purpose of this study was to present the case histories,

Correspondence to: Dr Shing Fung Lee, Department of Clinical Oncology, Tuen Mun Hospital, 23 Tsing Chung Koon Road, Tuen Mun, Hong Kong, SAR, P.R. China

E-mail: bestmic@gmail.com

Key words: colorectal cancer, antineoplastic drugs, adverse events pathophysiology, imaging findings and literature review for this uncommon condition.

\section{Case reports}

Case 1. A 61-year-old Chinese woman underwent laparoscopic right hemicolectomy for cancer of the ascending colon. On pathological examination, the tumor was diagnosed as moderately differentiated adenocarcinoma, stage pT4aN1aM0, KRAS wild-type. The patient completed 6 months of adjuvant capecitabine and oxaliplatin treatment, with good tolerance.

The carcinoembryonic antigen (CEA) levels increased, and a positron emission tomography/computed tomography (PET/CT) scan at 4 months after treatment revealed lung and liver metastases. The patient was treated with capecitabine and irinotecan (CAPIRI) and cetuximab (every 2 weeks). After 5 cycles, treatment was changed to CAPIRI and bevacizumab (every 2 weeks), as a follow-up PET/CT scan revealed disease progression. The patient exhibited a good clinical response, with a decrease in abdominal pain; the CEA level decreased from 82.1 to $18.4 \mathrm{ng} / \mathrm{ml}$. However, on day 9 of cycle 4 of CAPIRI and bevacizumab, the patient was admitted with right lower quadrant abdominal pain, watery diarrhea and vomiting. The patient also developed fever and the blood tests revealed neutropenia (nadir neutrophil count, $0.3 \times 10^{9} / 1$ ) and hypokalemia. The liver function tests were within normal limits. The differential diagnosis included infectious gastroenteritis aggravated by neutropenia and bevacizumab-related bowel perforation. The patient was treated conservatively with intravenous fluids, broad-spectrum antibiotics and granulocyte colony-stimulating factor (G-CSF). The fever subsided 1 day later and the neutrophil count increased to $>0.5 \times 10^{9} / 1$.

A CT scan of the abdomen and pelvis revealed extensive submucosal edema at the terminal and middle part of the ileum, associated with adjacent increase in fat stranding (Fig. 1). There was no pneumoperitoneum or bowel dilation. Evaluation was negative for sepsis, including blood, stool and urine cultures.

Hypokalemia persisted for 1 week and was managed with intravenous fluids and dietary modifications. The patient's condition gradually improved with conservative treatment, with improvement in oral intake and decreased vomiting and diarrhea. The patient remained in the hospital for a total of 12 days. The final diagnosis was terminal ileitis associated with capecitabine. 


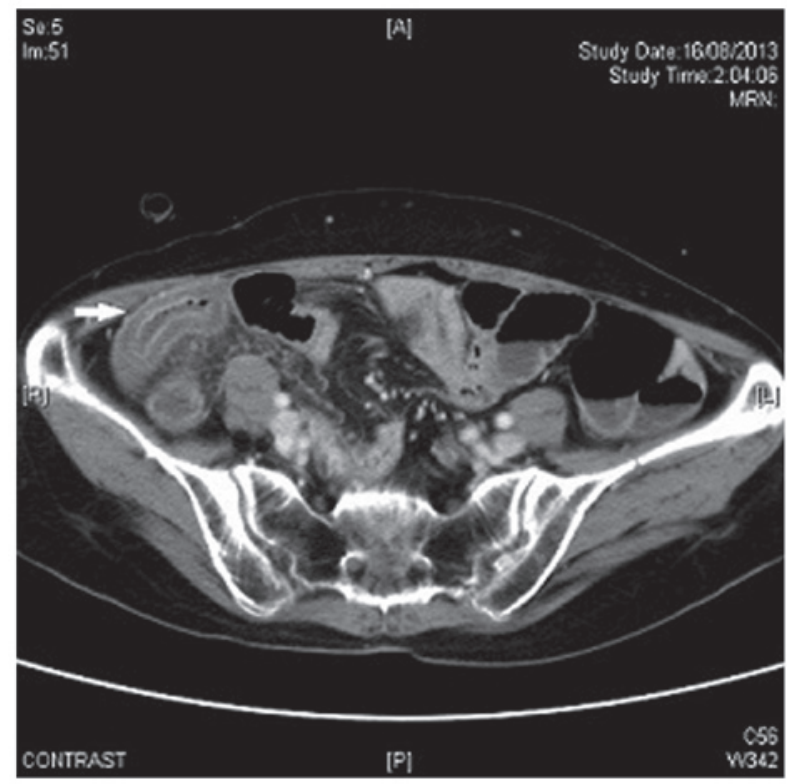

Figure 1. Case 1. Computed tomography scan. Axial view showing submucosal edema associated with adjacent increase in fat stranding. The bowe loops were not dilated.

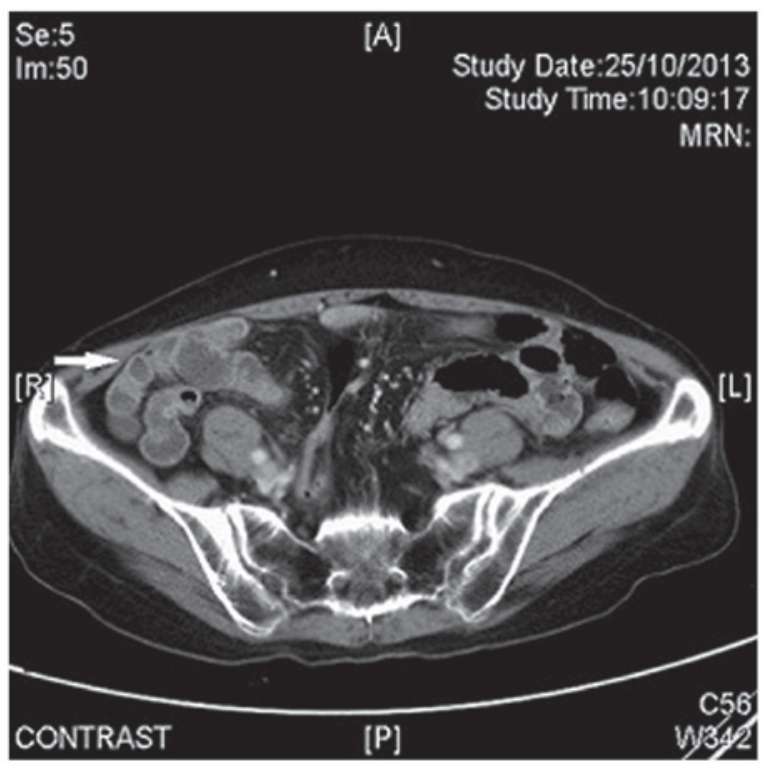

Figure 2. Case 1. Computed tomography scan. Axial view showing complete resolution of small bowel wall submucosal edema.

Chemotherapy with irinotecan and bevacizumab was resumed 1 week after hospital discharge, with no recurrence of ileitis. A CT scan performed 2 months later revealed complete resolution of the small bowel wall submucosal edema (Fig. 2).

Case 2. A 59-year-old Chinese woman underwent laparoscopic sigmoidectomy for sigmoid colon cancer. On pathological examination, the tumor was diagnosed as pT3NO adenocarcinoma with extramural vascular invasion. The patient was started on adjuvant capecitabine $\left(2,500 \mathrm{mg} / \mathrm{m}^{2} /\right.$ day orally on days 1-14 every 3 weeks) and exhibited good tolerance to chemotherapy during the first 2 cycles; however, she developed grade 1 diarrhea, hand-foot-skin reaction and stomatitis.

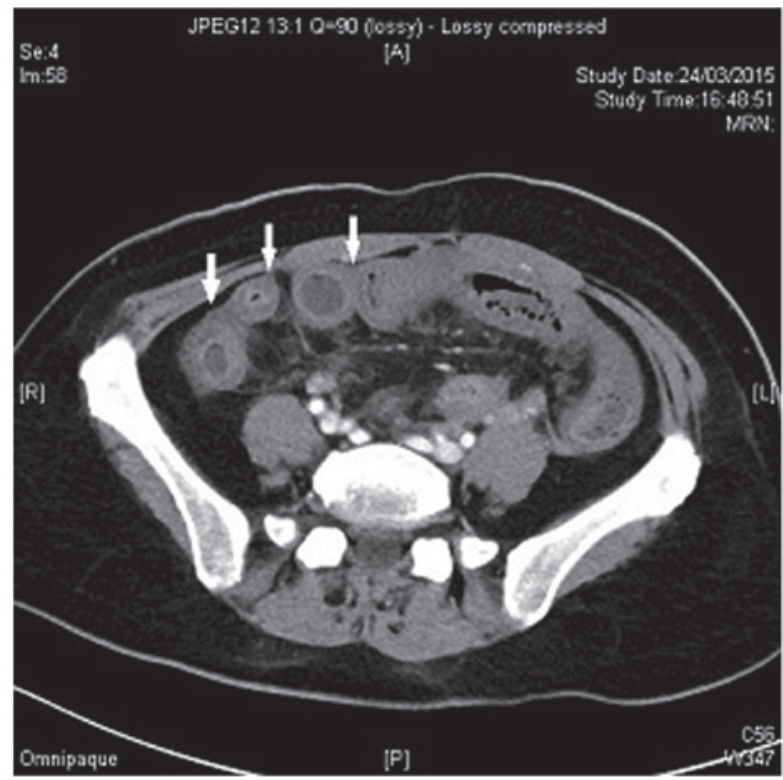

Figure 3. Case 2. Computed tomography scan. Axial view showing submucosal edema involving the distal to terminal ileum, with surrounding fat stranding, consistent with ileitis. Mucosal enhancement was preserved.

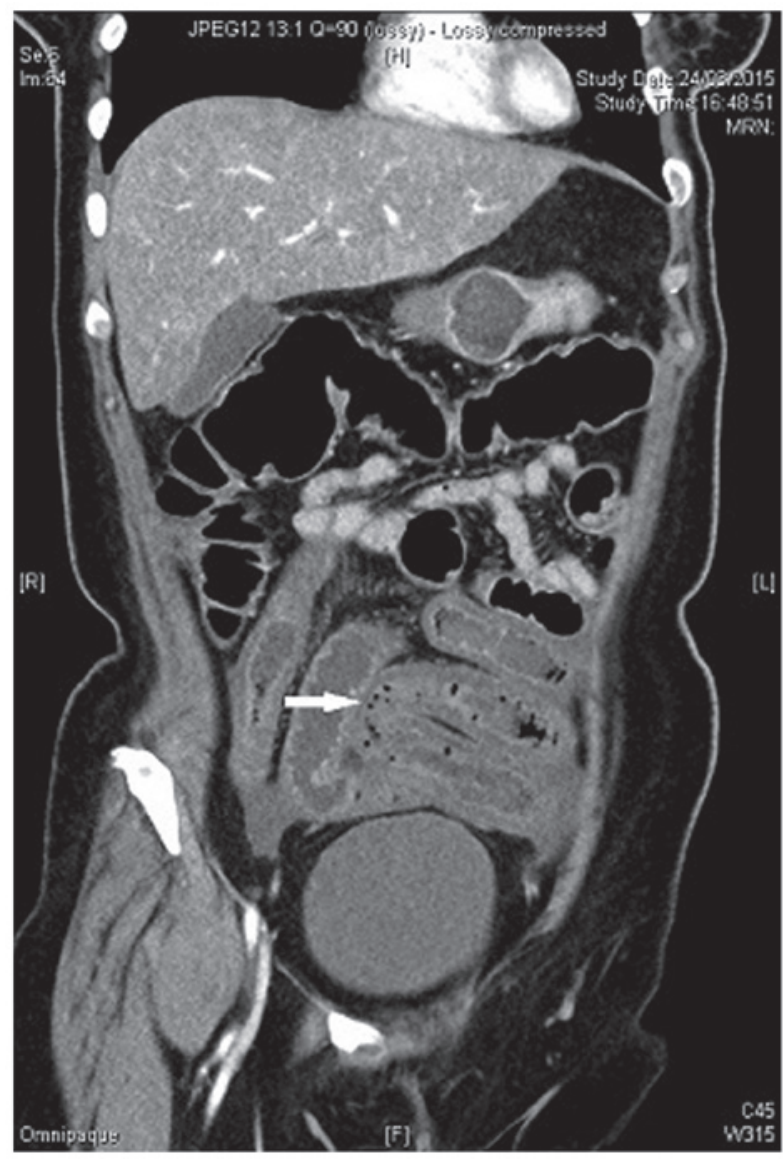

Figure 4. Case 2. Computed tomography scan. Coronal view showing multiple small gas bubbles along the edematous distal ileum, consistent with pneumatosis intestinalis.

After day 14 of cycle 3, the patient exhibited worsening diarrhea, and was admitted to the hospital on day 19 afterchemotherapy initiation; she had grade 3 mucositis, hand-foot-skin 
reaction, grade 4 diarrhea and severe generalized abdominal pain. A contrast-enhanced CT scan of the abdomen revealed diffuse submucosal edema with surrounding fat stranding, suggestive of ileitis in a long segment of the distal ileum to the terminal ileum. Multiple small gas bubbles were observed along the wall of the edematous distal ileum, consistent with pneumatosis intestinalis (Figs. 3 and 4).

The patient was admitted to the intensive care unit for close monitoring and received total parenteral nutrition due to diarrhea, hypoalbuminemia and decreased oral intake. Inotropic support and electrolyte replacement were given to maintain hemodynamic stability and avoid electrolyte disturbances.

The patient exhibited a decreased neutrophil count (minimum, $0.7 \times 10^{9} / 1$ on day 20 ) and developed fever on day 21. Evaluation was negative for sepsis, including blood, stool and urine cultures. The patient was treated with broad-spectrum antibiotics for a total of 10 days and G-CSF. The neutrophil count increased to $1.39 \times 10^{9} / 1$ after 2 days.

The patient's condition gradually improved and diarrhea subsided on day 29 after chemotherapy initiation. The mucositis and skin reaction resolved and parenteral nutrition was discontinued after 16 days.

\section{Discussion}

The two patients developed ileitis associated with capecitabine. Previously reported cases of capecitabine-associated ileitis included 1 patient who had received adjuvant capecitabine monotherapy for colon cancer, 1 patient who had received capecitabine, oxaliplatin and bevacizumab for metastatic rectal cancer, and 1 patient who had received capecitabine and oxaliplatin for metastatic rectal cancer (2-4). In these previous cases, the ileitis dveloped early, between the first and third cycle of chemotherapy, and none of the patients in these reports had previous exposure to capecitabine. Case 1 highlights the fact that small bowel complications may occur during later cycles of chemotherapy, despite previous good tolerance to capecitabine.

In both presented cases, it was difficult to confirm the diagnosis, as the initial clinical findings were non-specific, the patients were on combination chemotherapy plus targeted therapy, and this type of toxicity is rare. The CT scan was an important tool for diagnosis and monitoring. The radiographic findings were comparable in the two cases, and ileitis was observed as diffuse submucosal edema of the small bowel wall, which was consistent with previous case reports and confirmed the diagnosis.

It was important to closely monitor serum electrolyte levels during the acute phase of the ileitis. Adequate hydration was also important, as capecitabine is excreted primarily by the kidneys.

Capecitabine is administered as an oral prodrug; it is metabolized in successive enzymatic steps to 5-FU, which is metabolized into two active metabolites that exert the cytotoxic effect. The step of conversion to 5-FU preferentially occurs in tumor tissues (1). 5-FU may cause vasospasm in the coronary vessels and cardiotoxicity (5). Furthermore, the vascular endothelium is susceptible to 5-FU via the generation of free radicals. The endothelial damage may cause a procoagulant state and thrombosis $(6,7)$. These vascular effects of 5-FU may also affect intestinal vessels. A previous case of 5-FU-associated small bowel vasculitis due to arterial ischemia has been reported (8). Another study demonstrated that proinflammatory cytokines may contribute to 5-FU-associated intestinal mucosal injury (9).

In our cases, the contribution of bevacizumab and irinotecan to ileitis was unlikely. According to a previous case series, the most common clinical findings in bevacizumab-associated bowel perforation include abdominal pain and tenderness, vomiting, fever and leukocytosis. Diarrhea has been less frequently reported in most bevacizumab studies and is not a typical symptom of bowel perforation (10). A literature search identified no reported cases of small bowel ileitis associated with bevacizumab and irinotecan administration. The patient in case 1 did not have another episode of ileitis during subsequent cycles of irinotecan and bevacizumab treatment following discontinuation of capecitabine.

In summary, we presented two cases of adult Chinese patients who developed ileitis associated with capecitabine treatment. Ileitis may occur despite previous good tolerance to capecitabine. A high index of suspicion is crucial for prompt diagnosis. Ileitis may be reversible with early recognition, close monitoring and proper supportive treatment.

\section{References}

1. Saif MW, Katirtzoglou NA and Syrigos KN: Capecitabine: An overview of the side effects and their management. Anticancer Drugs 19: 447-464, 2008.

2. Radwan R, Namelo WC, Robinson M, Brewster AE and Williams GL: Ileitis secondary to oral capecitabine treatment? Case Rep Med 154981: 2012, 2012.

3. Bouma G and Imholz AL: Ileitis following capecitabine use. Ned Tijdschr Geneeskd 155: A3064, 2011. [In Dutch].

4. Al-Gahmi AM, Kerr IG, Zekri JM and Zagnoon AA: Capecitabine-induced terminal ileitis. Ann Saudi Med 32: 661-662, 2012.

5. Shoemaker LK, Arora U and Rocha Lima CM: 5-fluorouracil-induced coronary vasospasm. Cancer Contr 11: 46-49, 2004.

6. Jensen SA and Sørensen JB: 5-fluorouracil-based therapy induces endovascular injury having potential significance to development of clinically overt cardiotoxicity. Cancer Chemother Pharmacol 69: 57-64, 2012

7. Kuzel T, Esparaz B, Green D and Kies M: Thrombogenicity of intravenous 5-fluorouracil alone or in combination with cisplatin. Cancer 65: 885-889, 1990.

8. Bucaloiu ID, Dubagunta S, Pachipala KK, Kamal N and Fata F: Small-cell cancers, and an unusual reaction to chemotherapy: Case 4. Fluorouracil-related small bowel vasculitis. J Clin Oncol 21: 2442-2443, 2003.

9. Soares PM, Mota JM, Souza EP, Justino PF, Franco AX, Cunha FQ, Ribeiro RA and Souza MH: Inflammatory intestinal damage induced by 5 -fluorouracil requires IL-4. Cytokine 61: 46-49, 2013.

10. Badgwell BD, Camp ER, Feig B, Wolff RA, Eng C, Ellis LM and Cormier JN: Management of bevacizumab-associated bowel perforation: A case series and review of the literature. Ann Oncol 19: 577-582, 2008. 